\title{
Strates
}

STRATES Matériaux pour la recherche en sciences sociales

$11 \mid 2004$

Jeune recherche, la vitalité d'un laboratoire

\section{Paysages salicoles des marais charentais, essai de géographie historique et culturelle}

\author{
Sarah RÉAULT-MILLE
}

\section{(2) OpenEdition}

1 Journals

Édition électronique

URL : http://journals.openedition.org/strates/448

DOI : $10.4000 /$ strates. 448

ISSN : $1777-5442$

Éditeur

Laboratoire Ladyss

Édition imprimée

Date de publication : 1 janvier 2004

ISSN : 0768-8067

\section{Référence électronique}

Sarah RÉAULT-MILLE, « Paysages salicoles des marais charentais, essai de géographie historique et culturelle », Strates [En ligne], 11 | 2004, mis en ligne le 14 janvier 2005, consulté le 08 septembre 2020. URL : http://journals.openedition.org/strates/448; DOI : https://doi.org/10.4000/strates.448

Ce document a été généré automatiquement le 8 septembre 2020

Tous droits réservés 


\title{
Paysages salicoles des marais charentais, essai de géographie historique et culturelle
}

\author{
Sarah RÉAULT-MILLE
}

1 Thèse de géographie soutenue à l'université de Nantes le 18 décembre 2000 sous la direction de J.-P. Corlay, professeur des universités

2 Cette recherche avait pour objectif de comprendre l'évolution des rapports entre les sociétés littorales et les marais salicoles charentais. L'analyse paysagère a permis d'aborder l'évolution de la construction des marais, celle des rapports techniques et culturaux qui s'y sont succédés et des aménagements qui entretiennent le marais en tant que milieu producteur, puis, depuis la fin du $\mathrm{XX}^{\mathrm{e}}$ siècle, en tant que patrimoine naturel et/ou culturel. Le patrimoine s'exprimant aujourd'hui souvent sous la forme d'un paysage, cette étude locale permet aussi de tenter une réflexion conceptuelle sur le paysage dans les rapports changeants de l'homme à la terre.

Cette thèse se présente en trois parties. La première, "Paysage fossile? La strate structurelle du paysage, reflet des formes d'appropriation du marais salant ", prend le paysage comme archive de base pour se poser la question de l'évolution dans le temps long de la mise en place des marais charentais et de leur rôle dans la construction territoriale d'une marge littorale. Cette partie a permis de proposer une hypothèse de reconstitution de l'extension des marais salants entre un flou Haut Moyen Âge et le $\mathrm{XIX}^{\mathrm{e}}$ siècle; cette extension est contemporaine de la conquête du schorre. Elle correspond aussi à une extension territoriale fondamentale à deux niveaux : d'une part parce que dans le contexte d'un littoral très découpé, elle permet de relier des espaces auparavant isolés; d'autre part, parce qu'elle se situe dans un domaine de frontière littorale et politique et qu'elle en devient donc partie intégrante.

La deuxième partie, "Paysage mobile: la strate superficielle du paysage, reflet des usages modelant le marais salicole ", trace les évolutions techniques qui se portent sur ces marais. Elle se place entre une géographie historique et culturelle qui unit les sociétés à ces milieux amphibies, combinant pour produire trois ensembles d'éléments, 
air, terre, mer (conditions climatiques, édaphiques, maritimes). À des rythmes différents, chacun des marais voit se succéder plusieurs types d'appropriation. L'appropriation salicole est originelle, elle combine et maîtrise ces trois éléments pour produire du sel. Quand les rapports entre l'entretien et la sédimentation naturelle ne permettent plus l'irrigation en eau salée, le marais passe en marais doux utilisable par une agriculture qui réutilise un substrat déjà artificialisé (fin Moyen Âge au XX $\mathrm{XX}^{\mathrm{e}}$ siècle). Quand le marais est alimenté en eau salée, dans un cadre de déprise salicole (XIX ${ }^{\mathrm{e}}$-XX ${ }^{\mathrm{e}}$ siècle), les sociétés littorales favorisent la production ostréicole.

5 La troisième partie, « Paysages utiles, paysages futiles ? La strate culturelle du paysage, reflet des modes d'idéalisation du marais », cerne les aménagements qui ont permis de maintenir un marais productif et salubre et qui cherchent aujourd'hui, en grande partie, à en faire un lieu protégé, un patrimoine naturel et/ou culturel, progressivement construit en terroir de produits de qualité (huîtres, sel, bovins).

6 C'est sur les liens entre la deuxième et la troisième partie que nous insisterons ici pour montrer comment la succession des systèmes sociaux et techniques prend en charge un même milieu écologique, passant d'une dynamique dans laquelle l'entretien a pour but la production à long terme, le système productif, à une dynamique qui privilégie les formes protective et patrimoniale, le système protectif.

Un système productif : une protection intégrée à la production?

7 La saliculture se pratique dans les bassins artificiels creusés dans le sol argileux du marais, le bri, par une population paysanne, dans le cadre d'exploitations polyculturales. Il s'agit de maintenir une certaine quantité d'eau à l'intérieur de la saline et de l'évaporer progressivement dans trois bassins. Le réservoir est rempli au printemps, alors que le bassin de production est vidé de ses eaux douces et de ses vases accumulées pendant l'hiver. La saison productive commence au mois de juin quand le climat océanique permet une bonne évaporation éolienne et solaire. Il impose cependant aussi humidité et précipitations. L'activité nécessite donc une observation fine des conditions atmosphériques de façon à anticiper les manœuvres de gestion des eaux. Production et salubrité ne sont possibles que grâce au travail intensif d'une forte densité de population. Que la sédimentation ou que des crises économiques perturbent le système, l'État intervient. En cas d'échec, il y a déprise salicole, puis reprise sur un marais doux ou salé.

8 Sur les parties exondées du marais salant, les bosses, argileuses mais bien drainées, se pratiquait une culture de céréales; de même, certaines prairies naturelles, les sartières, sont pâturées. Après abandon, le marais gâtine, insalubre et non productif, est réaménagé. $\mathrm{Au} \mathrm{XVII}^{\mathrm{e}}$ comme au XIX ${ }^{\mathrm{e}}$ siècle, une recomposition du système hydraulique salicole permet le drainage des eaux douces et rend à nouveau les marais salubres et productifs. Dans les années cinquante, l'UNIMA (Union des Marais) prend en charge techniquement (gestion d'écluses) et socialement (syndicats de marais) cette gestion. L'élevage bovin est privilégié sur un marais inondé en hiver, humide au printemps, trop sec en été. Pour étendre au maximum la saison, l'éleveur compose avec les conditions hydropédologiques saisonnières. Les transformations lentes et anciennes du marais salicole en marais agricole ne permettent pas de savoir facilement si c'est la même population qui a repris l'entretien du marais.

9 L'élevage de coquillages se pratiquait dans les réservoirs et chenaux du marais salant. Entre le XIX ${ }^{e}$ siècle et le $\mathrm{XX}^{\mathrm{e}}$ siècle, les salines encore bien irriguées en eau salée sont transformées en claires à huîtres. Dans l'ostréiculture, la gestion de l'eau cherche à 
maintenir des niveaux d'eau suffisants pour conserver et affiner les coquillages. La saisonnalité, elle, repose non plus sur des rythmes climatiques, mais biologiques. Pour l'essentiel, la population ostréicole est issue de la reconversion d'anciennes familles de paysans-sauniers et de paysans. Jusqu'aux années soixante, ces exploitations gardent un aspect polycultural avant de se spécialiser.

Chacune des nouvelles activités a recomposé avec les éléments du milieu en fonction de ses besoins. L'agriculture se sépare d'une partie des apports marins, l'eau salée est écartée et seule une partie du sel nécessaire est maintenue. L'eau est maîtrisée, attirée en été, éloignée

aux intersaisons et l'agriculteur installe ses bêtes sur un marais préalablement assaini. L'ostréiculteur attire la mer et travaille l'eau pour affiner l'huître. Sur un même milieu, chacun compose selon ses besoins, chaque usage a sa propre interprétation du milieu, mais les éléments de base sont toujours là et leur présence modèle les subtilités techniques de chacun.

Du système protectif au système patrimonial et paysager

Dans les années quatre-vingt, la gestion du marais montre une opposition entre les producteurs et les protecteurs. La réglementation européenne suit et donne une image nouvelle des marais. La directive Oiseaux (1979) en fait un biotope pour les oiseaux classé en Zone de Protection Spéciale, protégée contre toute détérioration. Deux Réserves Naturelles sont créées sur des salines abandonnées. À la même période, naissent une aquaculture et une agriculture intensives. Ces nouvelles approches témoignent d'une séparation entre production/protection. Un premier système artificialise à l'extrême le milieu, l'autre valorise son aspect « naturel ».

Après le Sommet de la terre de Rio de Janeiro en 1992, cette opposition n'est officiellement plus antinomique. À l'échelle européenne, le traité de Maastricht et la nouvelle PAC associent environnement et agriculture. En 1992, la directive Habitats met en place le réseau écologique « Natura 2000 » pour lequel il est spécifié que la biodiversité est aussi induite par des activités humaines. Parallèlement, le paysage devient un objectif de développement local. Il prend place dans les Espaces Naturels Sensibles (1985), dans la loi Littoral de 1986 et est même directement exprimé par la loi Paysage de 1993. Qu'entraînent ces nouvelles approches dans la gestion des marais salicoles?

Dans la très touristique île de Ré, en 1995, 52 salines sont exploitées dans un marais en déprise totalement classé comme espace naturel. Une partie du monde agricole réagit en cherchant à y maintenir des activités primaires et une population permanente. Dans un programme de réhabilitation naît la notion de protection active. Deux à trois salines sont reprises par an. Les nouveaux systèmes de production privilégient la monoculture rendue possible par la revalorisation marchande d'un sel devenu symbole de nature et de tradition. La « machine salicole » est modifiée pour améliorer le rendement, limiter les déplacements. Les travaux de nettoyage étaient devenus plus rares ou moins soignés, ils sont aujourd'hui menés à la machine. Ce n'est pas toujours indispensable pour la production, mais il s'agit de remodeler un paysage devenu symbole d'un patrimoine collectif, naturel et culturel. Dans le marais agricole, l'élevage extensif est encouragé par des aides découlant des mesures agri-environnementales de la PAC et des Plans de Développement Durables qui demandent l'adaptation de certaines pratiques culturales aux «besoins" de la faune avicole, puis à la demande sociale d'environnement et de paysages. 
De même, longtemps peu touché par les systèmes protectionnistes, le marais ostréicole est aujourd'hui intégré à ces problématiques par l'intermédiaire de la probable construction

du réseau Natura 2000.

Nous avons vu que les systèmes d'exploitation traditionnels privilégient la production tout en assurant l'entretien permanent d'une machine hydraulique adaptée à chaque activité et imposée par le fonctionnement naturel du marais. L'entretien permanent est garant de la productivité. Nous sommes en quelque sorte dans un système de protection intégrée à la production. Dans le dernier tiers du XX $\mathrm{XX}^{\mathrm{e}}$ siècle, les évolutions techniques et culturelles entraînent de nouvelles formes de production maraîchines qui intègrent des formes d'intensification. L'intensification de la pression productive s'affirme sur chacun des milieux, tentant une sorte de séparation des contraintes qu'il impose à chacun des usages.

Dans cette logique, l'agriculture artificialise tous les éléments édaphiques, l'aquaculture intensive se sépare du sol et rythme ses propres besoins en eau. Enfin, alors qu'elle semble symboliser les idéaux d'une culture traditionnelle en symbiose avec le milieu de marais, la saliculture a ses propres formes d'intensification. La production pourrait être considérée comme une sorte de protection (et c'est d'ailleurs comme cela qu'elle est de plus en plus perçue), car reproduisant un milieu productif et salubre, mais les tendances protectionnistes conduisent à leurs propres formes d'intensification. L'entretien et le déploiement de moyens nécessaires à une forme de jardinage du milieu de marais ont leurs propres coûts en hommes, en temps et en argent. De même, ne peut-on pas parler d'intensification du regard esthétisant et paysager? Si le milieu de marais semble échapper selon ces regards aux pressions productivistes, elles-mêmes coûteuses et fragilisantes, c'est toujours au prix fort d'un déploiement de structures administratives, technocratiques et de subventions.

\section{INDEX}

Mots-clés : Paysage

Keywords : France 